\title{
Lorentz invariance, Unitarity and UV-finiteness of QFT on noncommutative spacetime
}

\author{
Anais Smailagic ††, Euro Spalluccił̦ \\ †Sezione INFN di Trieste, \\ Strada Costiera 11, 34014 Trieste, \\ Italy \\ $\ddagger$ Department of Theoretical Physics, \\ University of Trieste, Strada Costiera 11, 34014 Trieste, \\ Italy
}

\begin{abstract}
Ultraviolet finite quantum field theory on even dimensional noncommutative spacetime is formulated using coordinate coherent states. $2 d$ spacetime is foliated into families of orthogonal, non commutative, two-planes. Lorentz invariance is recovered if one imposes a single non commutative parameter $\theta$ in the theory. Unitarity is checked at the one-loop level and no violation is found. Being UV finite NCQFT does not present any UV/IR mixing.
\end{abstract}

PACS numbers: $11.10 . \mathrm{Nx}$

$\dagger$ e-mail address: anais@ictp.trieste.it

I e-mail address: spallucci@trieste.infn.it 
The interest in noncommutative geometry dates back to [1] where it was pointed out that Lorentz invariance does not exclude the existence of a fundamental length in the spacetime fabric. In this approach coordinates are lifted from mere spacetime labels to dynamical variables satisfying non-vanishing commutation relations. In order for some parameter to be given the role of "fundamental length" it has to be Lorentz invariant, i.e. must be inert under Lorentz boosts. If so, it can be safely interpreted as a new constant of nature representing the "minimal" distance having a physical meaning. The existence of a universal "quantum of length" should provide a natural answer to many different problems like curvature singularities in General Relativity and ultraviolet (UV) divergences in quantum field theory $(\mathrm{QFT})[2]$, [3].

Modern string theory re-invented the minimal length through introduction of a length invariant under $T$-duality transformations [4. It is quite remarkable that non-perturbative string dynamics introduces noncommutative $(\mathrm{NC})$ spacetime coordinates as well. It seems that there is a deep, yet not completely understood interplay among Lorentz invariance, noncommutativity (NCY) and the existence of a minimal length. Attempts to build up classical, or quantum, dynamics over a $\mathrm{NC}$ space(time) turns out to be a non-trivial task [5]. The most common way out of these difficulties is to replace NC coordinate operators with standard $c$-number coordinates and introduce a different multiplication rule between ordinary functions known as Wigner-Weyl-Moyal, or $*$-product, [6]. It has been originally used in the formulation of non-relativistic quantum mechanics (QM) in phase-space. Thus, based on the success in QM, it was a natural attempt to apply this formulation to quantum field theory as well. At this point, the problem of compatibility between Lorentz invariance and NCY becomes important, but we shall postpone its discussion. The output of application of the Moyal approach to QFT can be summarized into a deceptively simple prescription: take a commutative QFT and, "simply", replace the ordinary product by the $*$-product in the action. The quadratic terms in the action are not modified by the $*$-product since it adds only surface contributions. As a result only interaction terms are modified.

In spite of its apparent simplicity this prescription opened a "Pandora Box": the *product brings into NCQFT the intrinsic non-locality of the original NC geometry in the form of non-local interactions. This non-locality mimics the absence of "points" in the original $\mathrm{NC}$ geometry. On the other hand, the presence of non-local vertices introduces both technical and conceptual problems. On the technical side, the only way to perform calculations of measurable quantities, e.g. scattering amplitudes, it to expand the model in powers of the "theta-parameter" measuring the NCY of the original coordinates. At any finite order in "theta" the model is to all effects an ordinary local field theory ( though with additional vertices ), thus completely loosing the memory of its original non-locality. The resulting Feynman amplitudes have the same ultraviolet infinities as in the commutative case. Furthermore, new vertices introduce so-called "non-planar graphs" leading to an un-expected mixing between ultraviolet and infrared (IR) divergences. The conclusion is that a perturbative treatment on the Moyal-starred quantum field theory, not only is unable to get rid of the UV-infinities, as originally 
expected, but introduces a new kind of problem, i.e. the UV/IR mixing, in conflict with renormalization group philosophy.

Secondly, Lorentz invariance requires time to be included among the NC coordinates, but this may imply problems with unitarity [7]. Several different proposals have been advanced in order to deal with the clash between Lorentz invariance and unitarity varying from attempts to re-define time-ordering [8, to [9] abandoning Lorentz invariance, to those proposing "exotic" treatments such as to consider NCY as a sort of "stochastic" effect to be averaged over [10, [11], 12], [13], [14]. In our opinion, none of them offers neither totally convincing, nor simple solution. The skeptical reader can make his opinion by comparing, just to quote some, the conclusions in [15, [16], 1], [13].

Against this background, we proposed in [17, [18, an alternative formulation of NCY in terms of coherent states. Originally, our primary motivation was to cure short-distance behavior of the Feynman propagator along the reasoning related to the existence of a minimal length. An explicit computation of the vacuum expectation value of the energymomentum tensor has shown the infinity suppression mechanism at work [19]. We have shown that the coherent state approach naturally introduces a Gaussian cut-off in the Feynman propagator rendering NCQFT UV-finite. Our model was limited to $2+1$ dimensional spacetime, with only space non commutativity. Thus, though it did produce UV finite theory, it could not lead to Lorentz invariant propagator by construction. In this paper we would like to take on the previous results and improve them along two important directions outlined in the previous discussion:

first, formulate extension of coherent state approach to arbitrary dimensional spacetime. The intent is to discuss physically relevant four dimensional NCQFT.

Second, we want to obtain a Lorentz invariant and unitary NCQFT.

Let us start from the commutator for a set of $D$ hermitian operators $\hat{\mathbf{x}}^{\mu}, \mu=$ $1,2, \ldots D$

$$
\left[\hat{\mathbf{x}}^{\mu}, \hat{\mathbf{x}}^{\nu}\right]=i \hat{\theta}^{\mu \nu} \quad \mu, \nu=1,2 \ldots, D
$$

Commutator defined in (11) is taken as a starting point in almost all papers on NCY and all the problems raised depending on how one defines $\hat{\theta}^{\mu \nu}$. Usual choice of $\hat{\theta}^{0 i}=0$ led to a fixed direction in spacetime and to Lorentz non-invariance. In order to avoid problems of fixed directions in NC spacetime $\hat{\theta}^{\mu \nu}$ in (11) must be chosen as a Lorentz tensor. With such choice it is understood that time is also a NC coordinate, since it is the only way to hope for a final Lorentz invariant QFT. +

The assumption of Lorentz covariance of (1) is crucial. Then, one can exploit known property that any antisymmetric matrix can be brought by a suitable rotation to a block-diagonal form as

$$
\hat{\theta}^{\mu \nu}=\operatorname{diag}\left(\hat{\theta}_{1}, \hat{\theta}_{2} \ldots \hat{\theta}_{D / 2}\right)
$$

\footnotetext{
+ We shall work in Euclidean space-time where Lorentz symmetry is seen as (pseudo) rotations.
} 


$$
\hat{\theta}_{i} \equiv \theta_{i}\left(\begin{array}{cc}
0 & 1 \\
-1 & 0
\end{array}\right)
$$

In case of odd dimensional space(time) the last element on the diagonal is zero, thus in this space(time) there is always one coordinate commuting with all others. Covariance of (11) allows us to assert that apparent non-commutativity of all space-time coordinates is actually reducible to a set of non-commutative planes. In other words, $\mathrm{NC}$ spacetime can always be foliated in such a way that NCY is restricted to these privileged planes. Lorentz covariance of (11) means that different inertial observers see different non-commutativity of coordinates due to different projections of the $\mathrm{NC}$ planes in their respective frames. What they agree on is the existence of these planes in spacetime. In order to consider full $\mathrm{NC}$ we choose to work in even dimensional space times. In our picture, $D \equiv 2 d$ coordinates are represented by $d$ two-vectors $\overrightarrow{\hat{\mathbf{x}}}_{i}$ as

$$
\begin{aligned}
\hat{\mathbf{x}}^{\mu} & =\left(\hat{\mathbf{x}}^{1}, \hat{\mathbf{x}}^{2}, \ldots \hat{\mathbf{x}}^{2 d-1} \hat{\mathbf{x}}^{2 d}\right) \\
& =\left(\overrightarrow{\hat{\mathbf{x}}}_{1}, \ldots \overrightarrow{\hat{\mathbf{x}}}_{d}\right)
\end{aligned}
$$

where $\overrightarrow{\hat{\mathbf{x}}}_{i} \equiv\left(\hat{\mathbf{y}}_{1 i}, \hat{\mathbf{y}}_{2 i}\right)$ are two-vectors with $\left(\hat{\mathbf{y}}_{1 i}, \hat{\mathbf{y}}_{2 i}\right)$ coordinates of the $i$-th NC two-plane satisfying

$$
\left[\hat{\mathbf{y}}_{1 i}, \hat{\mathbf{y}}_{2 i}\right]=i \theta_{i}
$$

Thus, the problem is reduced to an effective two-dimensional one. Pioneering approach in use of coherent states regarded quantum optics and referred to phasespace. Coherent states are defined as eigenstates of properly defined ladder operators [20]. As already stated, the reason behind use of coherent states is that there are no coordinate eigenstates for $\mathrm{NC}$ coordinates and no coordinate representation can be defined. Therefore, ordinary wave functions (in QM) or fields defined over points (in QFT) can not be defined anymore. Coherent states are the closest to the sharp coordinate states that one can define for $\mathrm{NC}$ coordinates in the sense that they are minimal-uncertainty states and enable, in spite of absence of sharp coordinate eigenvalues, to define mean values of coordinate operators.

In order to apply the coherent state approach we have to build appropriate set of mutually commuting ladder operators built from NC spacetime coordinates only. This point is important to stress since often there is a confusion among readers between coherent states in phase space and our coherent states in NC coordinate space-time. Ladder operators should satisfy usual commutation rules of creation and destruction operators of QM. Mean values of any operator over coherent states are commutative quantities upon which one can construct the commutative QFT. In block-diagonal basis of (11) we define ladder operators of the $i$-th plane as

$$
a_{i}=\frac{1}{\sqrt{2}}\left(\hat{\mathbf{y}}_{1 i}+i \hat{\mathbf{y}}_{2 i}\right)
$$




$$
a_{i}^{\dagger}=\frac{1}{\sqrt{2}}\left(\hat{\mathbf{y}}_{1 i}-i \hat{\mathbf{y}}_{2 i}\right)
$$

The ladder operators satisfy usual canonical commutation rules:

$$
\left[\mathbf{a}_{i}, \mathbf{a}_{j}^{\dagger}\right]=\delta_{i j} \theta_{i}
$$

Normalized coherent states, $\langle\alpha \mid \alpha\rangle=1$ for the above ladder operators, are defined as

$$
|\alpha\rangle \equiv \prod_{i} \exp \left[\frac{1}{\theta_{i}}\left(\bar{\alpha}_{i} \mathbf{a}_{i}-\alpha_{i} \mathbf{a}_{i}^{\dagger}\right)\right]|0\rangle
$$

where, $|0\rangle$ is the vacuum state annihilated by $\mathbf{a}_{i}$.

The basic ingredient of our approach is to associate commutative coordinates to NC ones as their mean values over coherent states, much in the same way in which one relates classical and quantum variables in Quantum Mechanics [21]. Thus, mean coordinates are

$$
\begin{aligned}
\left\langle\alpha\left|\hat{\mathbf{y}}_{1 i}\right| \alpha\right\rangle & =\sqrt{2} \operatorname{Re} \alpha_{i} \\
\left\langle\alpha\left|\hat{\mathbf{y}}_{2 i}\right| \alpha\right\rangle & =\sqrt{2} \operatorname{Im} \alpha_{i}
\end{aligned}
$$

We associate ordinary functions of mean coordinates to operator-valued functions through their mean values as follows: $\langle\alpha|F(\hat{\mathbf{x}})| \alpha\rangle \equiv F(\alpha)$. Now, we can define a $\mathrm{NC}$ version of the Fourier transform as

$F(\alpha)=\int \prod_{i=1}^{d}\left(\frac{d \vec{p}_{i}}{2 \pi}\right) f\left(\vec{p}_{1}, \ldots \vec{p}_{d}\right)\left\langle\alpha\left|\exp \left[i \sum_{i=1}^{d}(\vec{p} \cdot \overrightarrow{\hat{\mathbf{x}}})_{i}\right]\right| \alpha\right\rangle$

The novel ingredient of the NC Fourier transform is the mean plane wave : $\langle\alpha|\exp (i \vec{p} \cdot \overrightarrow{\hat{\mathbf{x}}})| \alpha\rangle$. At this point it is important to stress the difference between our approach and the usual $*$-product. In our approach the product among functions of mean coordinates is not modified, rather mean values of operator valued functionals between NC coordinate coherent states makes NCY visible already at the level of a single function or field. This will turn out to be instrumental in obtaining UV finite NCQFT, as we shall see later. The explicit form of a NC plane wave can be calculated, using Hausdorff decomposition, as

$$
\left\langle\alpha\left|\exp \left[i \sum_{i=1}^{d}(\vec{p} \cdot \overrightarrow{\hat{\mathbf{x}}})_{i}\right]\right| \alpha\right\rangle=\exp \left[-\sum_{i=1}^{d}\left(\frac{1}{4} \theta_{i} \vec{p}_{i}^{2}+i(\vec{p} \cdot \vec{y})_{i}\right)\right]
$$

where, $\vec{y}_{i}=\left(\Re \alpha_{i}, \Im \alpha_{i}\right)$ is the mean position vector in the $i$-th plane. The NC plane wave (13) has been also used in formulating the $\mathrm{NC}$ version of the path integral. We shall now calculate the Feynman propagator in the mean value formalism using the prescription of [17] 


$$
\begin{aligned}
& G\left(\vec{y}_{1}-\vec{y}_{1}^{\prime}, \vec{y}_{2}-\vec{y}_{2}^{\prime} \ldots, \vec{y}_{d}-\vec{y}_{d}^{\prime} ; m^{2}\right) \\
& \equiv N \int[D e] \prod_{i=1}^{d}\left[D y_{i}\right] \prod_{j=1}^{d}\left[D p_{j}\right] \times \\
& \exp \left\{i \sum_{i=1}^{d}\left(\int_{y}^{y^{\prime}} \vec{p} \cdot d \vec{y}\right)_{i}-\right. \\
& \left.\int_{0}^{T} d \tau\left[e(\tau)\left(\sum_{i=1}^{d} \vec{p}_{i}^{2}+m^{2}\right)+\frac{1}{2 T} \sum_{i=1}^{d} \theta_{i} \vec{p}_{i}^{2}\right]\right\}
\end{aligned}
$$

Direct calculation leads to the final form of the Feynman propagator

$$
\begin{aligned}
& G\left(\vec{y}_{1}-\vec{y}_{1}^{\prime}, \vec{y}_{2}-\vec{y}_{2}^{\prime} \ldots, \vec{y}_{d}-\vec{y}_{d}^{\prime} ; m^{2}\right) \\
& =\int \prod_{i=1}^{d}\left(\frac{d \vec{p}_{i}}{(2 \pi)^{2}}\right) e^{i \sum_{i=1}^{d}\left[\vec{p} \cdot\left(\vec{y}-\vec{y}^{\prime}\right)\right]_{i}} G_{\theta}\left(\vec{p}_{1}, \ldots, \vec{p}_{d} ; m^{2}\right)
\end{aligned}
$$

where, $G\left(\vec{p}_{1}, \ldots, \vec{p}_{d} ; m^{2}\right)$ is the momentum space propagator, given by

$$
G\left(\vec{p}_{1}, \ldots, \vec{p}_{d}\right)=\frac{1}{(2 \pi)^{2 d}} \frac{1}{\sum_{j=1}^{d} \vec{p}_{j}^{2}+m^{2}} \exp \left[-\frac{1}{2} \sum_{i=1}^{d} \theta_{i} \vec{p}_{i}^{2}\right]
$$

As anticipated above, the use of mean values of coherent states leads to the exponential cut-off in the Green function of NCQFT which makes it UV finite. At this point it is important to investigate Lorentz invariance of this result. We see that the denominator is already expressed in terms of Lorentz invariant length of four momentum. On the other hand, Lorentz invariance of the exponential term is not so obvious because parameters $\theta_{i}$ are coupled to two-vectors. Thus, one should rewrite the exponent, in terms of $\Theta^{\mu \nu}$ tensor, in order to discuss invariance properties of the Green function. To clarify this point, let us consider the following combination which is a Lorentz scalar: $\delta_{\mu \lambda} \hat{\theta}^{\mu \nu} p_{\nu} \hat{\theta}^{\lambda \rho} p_{\rho}$. In the basis where $\hat{\theta}^{\mu \nu}$ assumes block-diagonal form, $\hat{\theta}^{\mu \nu}$ acts as a projector on the planes where the NCY is non-zero. If we define the projected momentum as $\tilde{p}^{\mu} \equiv \hat{\theta}^{\mu \nu} p_{\nu}$, then

$$
\begin{aligned}
\hat{\theta}^{\mu \nu} p_{\nu} \hat{\theta}_{\mu}^{\rho} p_{\rho} & =\tilde{p}^{2} \\
& =\sum_{i=1}^{d} \theta_{i}^{2} \vec{p}_{i}^{2}
\end{aligned}
$$

Comparison between (17) and (16) shows that in the latter $\theta_{i}$ enter in a noncovariant way. On the other hand, in the same basis the determinant of the matrix $\hat{\theta}^{2}$ is given by

$$
\operatorname{det}\left[\hat{\theta}^{\mu \nu} \hat{\theta}_{\mu}^{\rho}\right]=\prod_{i=1, \ldots, d} \theta_{i}^{2}
$$


We define an effective metric $\kappa^{\mu \nu}$ as

$$
\left[\operatorname{det}\left(\hat{\theta}^{\mu \nu} \hat{\theta}_{\mu}^{\rho}\right)\right]^{-1 / 4 d} \hat{\theta}^{\mu \nu} \hat{\theta}_{\mu}^{\rho} \equiv \kappa^{\nu \rho}
$$

$\kappa^{\mu \nu} p_{\mu} p_{\nu} \neq \sum_{i=1}^{d} \theta_{i} \vec{p}_{i}^{2}$ which stands in the exponent of (16). However, if we assume that the NC parameters $\theta_{i}$ in different planes are all equal, i.e. $\theta_{i} \equiv \theta$, then

$$
\left[\operatorname{det} \hat{\theta}^{\mu \nu} \hat{\theta}_{\mu}^{\rho}\right]^{-1 / 4 d} \hat{\theta}^{\mu \nu} \hat{\theta}_{\mu}^{\rho} p_{\nu} p_{\rho}=\kappa^{\nu \rho} p_{\nu} p_{\rho}=\theta \delta^{\mu \nu} p_{\mu} p_{\nu}
$$

and the propagator becomes Lorentz invariant. The choice of coincident $\theta_{i}$ gives the form of the effective metric as

$$
\kappa^{\mu \nu}=\theta \delta^{\mu \nu}
$$

The same choice has been implemented as an ansatz in [16], 27]. Here, we show how it can be constructed using coherent states. Thus, Lorentz invariance and UV finiteness of NCQFT is assured assuming a unique parameter $\theta$ expressing the spacetime NCY. Regarding UV finiteness, it is important to remark that the Gaussian cut-off is the intrinsic ingredient of coherent states NCQFT and not an ad hoc regularization device. The outcome of the above discussion is Lorentz invariant Feynman propagator

$$
G\left(p^{2}\right)=\frac{1}{(2 \pi)^{2 d}} \frac{1}{p^{\mu} p_{\mu}+m^{2}} \exp \left[-\frac{1}{2} \theta p^{\mu} p_{\mu}\right]
$$

Now we shall describe one of the important effect of NCY by considering the equation satisfied by the Green function in (mean)coordinate space. Direct calculation, using (22) gives

$$
\left[-\partial^{2}+m^{2}\right] G(x-y)=\frac{1}{(2 \pi \theta)^{2}} \exp \left[-\frac{(x-y)^{2}}{2 \theta}\right]
$$

We see that the effect of NCY in the space of commutative mean coordinates amounts to the substitution of Dirac delta-function ( point-like source) by a Gaussian function ( smeared source). Once again the NCY has produced an extension of all point-like structures to smeared ones. It is a natural consequence of the use of mean values to define spacetime positions.

Now we address the largely debated, and controversial, question of violation of unitarity in physical amplitudes of NCQFT. This problem was originally raised in [7] and further discussed in [13] with contradictory conclusions. Various cures to this problem have been proposed [25], 28], 29], again with controversial conclusions.

In order to address this problem in our approach, and for the reader to be able to compare it to previous attempts, we consider a two-point amplitude in scalar $\phi^{3}$ field theory as in [7]. Our calculation is based on the Feynman propagator (22). Consider the two-point amplitude given by

$\Gamma_{4}(p)=\int \frac{d^{4} q}{(2 \pi)^{4}} \frac{e^{-\frac{\theta}{2} q^{2}}}{q^{2}+m^{2}} \frac{e^{-\frac{\theta}{2}(p+q)^{2}}}{(p+q)^{2}+m^{2}}$ 
Explicit calculations in QFT rely on a widely adopted method which is the Schwinger proper-time representation of Feynman propagator. Due to the Gaussian cut-off this prescription has to be suitably modified. We find that the Schwinger representation of (22) is

$$
\frac{e^{-\frac{\theta}{2} q^{2}}}{q^{2}+m^{2}}=e^{\theta m^{2} / 2} \int_{\theta / 2}^{\infty} d s \exp \left[-s\left(q^{2}+m^{2}\right)\right]
$$

Representation (25) is crucial for greatly simplifying the actual calculation of any Feynman amplitude. With prescription (25), amplitude (24) becomes

$$
\Gamma_{4}(p)=e^{\theta m^{2}} \int_{\theta / 2}^{\infty} d s \int_{\theta / 2}^{\infty} d t \int \frac{d^{4} q}{(2 \pi)^{4}} \exp \left[-s\left(q^{2}+m^{2}\right)-t\left((p+q)^{2}+m^{2}\right)\right]
$$

Upon integrating internal momentum $q(24)$ is recast into

$$
\Gamma_{4}(p)=\frac{1}{16 \pi^{2}} e^{\theta m^{2}} \int_{\theta / 2}^{\infty} \int_{\theta / 2}^{\infty} \frac{d s d t}{(s+t)^{2}} \exp \left[-(s+t) m^{2}-p^{2} \frac{s t}{s+t}\right]
$$

Introducing a change of variables $s=(1-x) \lambda$ and $t=x \lambda$ one obtains

$$
\begin{aligned}
& \Gamma_{4}(p)=\frac{1}{16 \pi^{2}} e^{\theta m^{2}} \int_{0}^{1} d x \int_{\theta\left(m^{2}+x(1-x) p^{2}\right)}^{\infty} \frac{d \lambda}{\lambda} e^{-\lambda} \\
& =-\quad \frac{1}{16 \pi^{2}} e^{\theta m^{2}} \int_{0}^{1} d x \operatorname{Ei}\left[-\theta\left(m^{2}+x(1-x) p^{2}\right)\right]
\end{aligned}
$$

where $\operatorname{Ei}\left[-\theta\left(m^{2}+x(1-x) p^{2}\right)\right]$ is the Exponential Integral. One can make use of the explicit expression for $E i(x)$ to find the useful form of the amplitude as

$$
\begin{aligned}
\Gamma_{4}(p)=\quad & -\frac{1}{16 \pi^{2}} e^{\theta m^{2}}\left\{\gamma+\int_{0}^{1} d x \ln \left[\theta\left(m^{2}+x(1-x) p^{2}\right)\right]\right. \\
& \left.+\sum_{n=1}^{\infty} \frac{(-1)^{n}}{n n !} \int_{0}^{1} d x \theta^{n}\left(m^{2}+x(1-x) p^{2}\right)^{n}\right\}
\end{aligned}
$$

Now we are ready to investigate the question of unitarity (non)violation in our framework. As a brief reminder, the unitarity of physical amplitudes follows from the probabilistic interpretation of quantum mechanics and is encoded into the Optical Theorem

$$
2 \operatorname{Im} \Gamma_{a b}=\sum_{n} \Gamma_{a n} \Gamma_{n b}^{\dagger}
$$

where the sum is over all possible intermediate states.

Problem with unitarity in physical amplitude may arise if the above relation is not satisfied.

In our case the amplitude in the l.h.s. of (30) is given by (29), while the r.h.s. corresponds to the tree-level, on-shell, decay of a momentum $p$ scalar particle into 
two particles of the same kind. All calculations, so far, have been done in Euclidean spacetime. This is our general prescription to perform virtual momentum integrations in Euclidean spacetime, where the exponential cutoff has unambiguous sign. Only the final results are, then, continued into Minkowski spacetime and the physical implications are discussed. Thus, in order to investigate unitarity we Wick rotate (29) back to real time.

The imaginary part in (29) arises only from the logarithm due to its cut along the real axis. There are two physical regions of momentum to be considered, i.e. space-like $\left(p^{2}>0\right)$ and time-like $\left(p^{2}<0\right)$ momenta. First we consider space-like momenta. In this kinematical region there is no imaginary part in (29) since the argument of the logarithm never becomes negative in the integration region of $x$. Thus, for space-like momentum (30) is satisfied since the r.h.s. is also zero due to the energy-momentum conservation. Unitarity violation in the space-like region does not take place.

To complete the analysis of the unitarity non-violation we shall check the relation (30) also in the time-like region by performing an explicit calculation of both r.h.s. and l.h.s. in (30).

For time-like momentum we have $p^{2}=-\left|p^{2}\right|$, and the logarithm has an imaginary part for negative values of its argument $m^{2}-x(1-x)\left|p^{2}\right|$. This is true for the following values of the integration variable $x$ :

$$
\begin{aligned}
& x_{1} \leq x \leq x_{2} \\
& x_{1}=\frac{1-a}{2} \\
& x_{2}=\frac{1+a}{2} \\
& a \equiv \sqrt{1-\frac{4 m^{2}}{\left|p^{2}\right|}}
\end{aligned}
$$

Thus, for time-like momenta one finds imaginary part of the l.h.s. of (29) to be

$$
\operatorname{Im} \Gamma_{4}(p)=-\frac{1}{16 \pi} e^{\theta m^{2}} \sqrt{1-\frac{4 m^{2}}{\left|p^{2}\right|}}
$$

which gives the same result as in the commutative case, aside the exponential factor keeping track of the NCY. Notice that (35) is of particularly simple form compared to the same amplitude computed in various approaches within the $*$-product formulation. The commutative limit of (35) is immediately obtained by letting $\theta \rightarrow 0$.

To complete the check of unitarity we shall calculate the tree level diagram, i.e. the r.h.s. of (301). The way to perform this calculation is to use cutting rules in the (29). Cutting rules correspond to the substitution of internal propagators within the Feynman graph by a mass-shell delta-function * which extracts the imaginary part of the matrix

* To avoid misunderstanding, we stress that Dirac delta-functions are still present in the momentum space since the momenta are commuting from the start. It is only in position space that Dirac deltafunctions are replaced by Gaussian functions. 
element according to the prescription:

$$
\frac{1}{q^{2}+m^{2}} \longrightarrow-2 \pi i \delta\left(q^{2}+m^{2}\right)
$$

Thus, $\Gamma_{4}(p)$ turns out to be

$$
\Gamma_{4}^{\text {tree }}=-\frac{4 \pi^{2}}{(2 \pi)^{4}} e^{\theta m^{2}} \int d^{4} q \delta\left(p^{2}+m^{2}\right) \delta\left[(p+q)^{2}+m^{2}\right]
$$

We exploit the fact that $p^{2}$ is time-like to choose the rest-frame: $\vec{p}=0$. Lorentz invariance of the amplitude, in our approach, guarantees that the result obtained in the rest-frame will hold in any inertial frame. The calculation is performed by first integrating over $q_{0}$ component of internal momentum and then over $\vec{q}$, leading to

$$
\begin{aligned}
\Gamma_{4}^{\text {tree }} & =-\frac{1}{16 \pi^{2}} e^{\theta m^{2}} \int d^{3} q \frac{1}{2\left|p_{0}\right|} \delta\left[\vec{q}^{2}+m^{2}-\frac{p_{0}^{2}}{4}\right] \\
& =-\frac{1}{8 \pi} e^{\theta m^{2}} \sqrt{1-\frac{4 m^{2}}{\left|p^{2}\right|}}
\end{aligned}
$$

In the (38) we have reconstructed the explicitly Lorentz invariant form by taking into account that in the rest-frame $\left|p^{2}\right|=p_{0}^{2}$. Comparison between (38) and (35) shows that the unitarity condition (30) holds also for time-like momentum. Our conclusion is that there is no unitarity violation in NCQFT based on coherent state approach.Our statement obviously refers to the model considered and to one-loop calculations. Further investigation of the question of unitarity non-violation in more complicated models and at higher loop level are required in order to confirm this preliminary result on general grounds. Nevertheless, in spite of its limitations this is the first time that the absence of unitarity problem is proven in a model of NCQFT. We intend to further study this problem in future papers.

We give the summary of results on the NCQFT presented in this paper. What emerges as a direct consequence of the use of coherent states is a momentum Gaussian cutoff suppressing UV divergences. Resulting UV finiteness can be traced back to the existence of "minimal length" as an intrinsic property of the NC spacetime geometry. In coordinate space this property manifests itself in an equally simple form: the substitution of coordinate Dirac delta-functions with Gaussian functions. This is the reason why we believe that NCY effects should be always seen, thus even at the level of free Feynman propagator.

We have also shown that Lorentz invariant results can be obtained if one assumes a single parameter $\theta$. In this way, not only one obtains Lorentz invariant theory, but also reduces to minimum the number of free parameters in the model. This is in agreement with the "philosophy of simplicity" which requires the minimal arbitrariness. Same conclusions, though on the basis of quantum gravity arguments, regarding a single NC parameter are present in [22], 23], 24].

Finally, we have investigated very important issue of unitarity in NCQFT in view of 
previous inconclusive discussions. There have been attempts to attribute the lack of unitarity in NCQFT as their being an "incomplete" approximation with respect to string theory. Our philosophy in this paper is slightly different because NCY in QFT has been introduced much before string theory, [1, and with different motivations. Therefore, our interest was to investigate the extension of commutative QFT to its $\mathrm{NC}$ version independently from the origin of NCY itself. We have proven that this extension can be Lorentz invariant and unitary within the limits of the present model. We calculated explicitly a one-loop Feynman amplitude for $\phi^{3}$ scalar NCQFT and proved that unitarity is preserved. Contrary to the complicated analysis in the $*$-product approach, our calculation is particularly simple and transparent thanks to the modification of the Schwinger proper-time representation of the Feynman propagator (25). This modification enable us to follow as close as possible same calculations in the commutative case. The procedure offers no ground to believe that there should be any departure form one-loop conclusions at higher perturbative order, but this point requires further investigation.

Recently an approach using smeared fields [25], 26], [27, and based on earlier ideas presented in 24], has led to the same conclusion regarding the absence of UV/IR mixing and finiteness.

Although we agree with those results, there is a slight difference in underlying philosophy. The smearing proposed in [24] and followed in [27], is an ansatz motivated by quantum gravity arguments, while in our approach it is a direct result of the application of coherent state mean values. Furthermore, mentioned authors propose to put the smearing only in the interaction terms while keeping the kinetic term unchanged. From our point of view, NCY is a geometrical property of spacetime and is independent of the particular interactions of matter fields. To put it "boldly", a single car ( free particle ) traveling on the cobbled road ( $\mathrm{NC}$ spacetime) feels the bumps without need to crash (interact) into another car.

\section{Acknowledgments}

We would like to thank Prof.T.Padmanabhan for reading the draft of this note, and for useful discussion on the related issue of zero-point length. 


\section{References}

[1] H. S. Snyder Phys. Rev. 7138 (1947)

[2] T. Padmanabhan, Class. Quant. Grav. 4 (1987), L107; T. Padmanabhan String theory and the origin of zero point length to space-time, (Tata Inst.). Print-88-0033 (TATA), (1988), KEY 1776797; T. Padmanabhan, Phys. Rev. Lett.78 (1997), 1854

[3] A. Smailagic, E. Spallucci, T. Padmanabhan String theory T-duality and the zero point length of spacetime; hep-th/0308122

[4] M. B. Green, J. H. Schwartz, L. Brink Nucl. Phys. B198, 474 (1982)

K. Kikkawa, M. Yamasaki Phys. Lett. B149, 357 (1984)

N. Sakai, I. Senda Progr. Theor. Phys. 75, 692 (1986)

V. P. Nair, A. Shapere, A. Strominger, F. Wilczek Nucl. Phys. B287, 402 (1987)

B.Sathiapalan Phys. Rev. Lett. 58, 1597 (1987)

P. Ginsparg, C. Vafa Nucl. Phys. B289, 414 (1987)

A. Sen Int.J.Mod.Phys. A9 3707 (1994)

[5] L. Alvarez-Gaume', S. R. Wadia Phys. Lett. B501 (2001) 319

[6] H. Weyl Z.Phys. 461 (1927);

E. P. Wigner Phys. Rev.40 749 (1932);

G. E. Moyal Proc. Camb. Phyl. Soc. 4599 (1949)

[7] J. Gomis, T. Mehen Nucl.Phys. B591 (2000) 265

[8] H. Bozkaya, P. Fischer, H. Grosse, M. Pitschmann, V. Putz, M. Schweda, R. Wulkenhaar Eur. Phys. J. C29 (2003) 133

[9] S. M. Carroll, J. A. Harvey, V. A. Kostelecky, C. D. Lane, T. Okamoto Phys. Rev. Lett. 87 (2001) 141601

[10] C. E. Carlson, C. D. Carone, N. Zobin Phys. Rev. D66 (2002) 075001

[11] K. Morita Prog.Theor.Phys. 108 (2003) 1099

[12] H. Kase, K. Morita, Y. Okumura, E. Umezawa Prog. Theor. Phys. 109 (2003) 663

[13] K. Morita, Y. Okumura, E. Umezawa Prog.Theor.Phys. 110 (2003) 989

[14] K. Morita New Gauge-Invariant Regularization Scheme Based on Lorentz-Invariant Noncommutative Quantum Field Theory; hep-th/0312080

[15] M. Chaichian, A. Demichev, P. Presnajder Nucl.Phys. B567 (2000) 360

[16] S. Cho, R. Hinterding, J. Madore, H. Steinacker Int. J. Mod. Phys. D9 (2000) 161

[17] A. Smailagic, E. Spallucci J.Phys.A36 (2003) 467

[18] A. Smailagic, E. Spallucci J.Phys.A36 (2003) 517

[19] P. Nicolini Vacuum energy momentum tensor in (2+1) NC scalar field theory hep-th/0401204

[20] R. J. Glauber Phys. Rev.131 2766 (1963)

[21] A. Messiah Quantum Mechanics, North Hollad Publ. Comp. (1961)

[22] S. Doplicher, K. Fredenhagen, J. E. Roberts Commun. Math. Phys. 172 (1995) 187

[23] D. Bahns, S. Doplicher, K. Fredenhagen, G. Piacitelli Phys.Lett. B533 (2002) 178

[24] D. Bahns, S. Doplicher, K. Fredenhagen, G. Piacitelli Commun. Math. Phys. 237 (2003) 221

[25] P. Fischer, V. Putz Eur. Phys. J. C32 (2004) 269

[26] S. Denk, V. Putz, M. Schweda, M. Wohlgenannt Towards UV Finite Quantum Field Theories from Non-Local Field Operators; hep-th/0401237

[27] P. Fischer, V. Putz No UV/IR mixing in unitary spacetime noncommutative field theory, hep-th/0306099

[28] Y. Liao, K. Sibold Eur. Phys. J. C25 (2002) 479

[29] T. Ohl, R. Rckl, J. Zeiner Nucl.Phys. B676 (2004) 229 